\title{
Probing the subtleties of atomic distortions through accurate STEM imaging and density functional theory
}

\author{
James LeBeau, Xiahan Sang, J. Houston Dycus, Changning Nui, Douglas Irving \\ Department of Materials Science \& Engineering, North Carolina State University, Raleigh, NC USA
}

While aberration correction has unlocked previously inaccessible information at the atomic scale, realspace measurements of atomic displacements and distances have remained largely qualitative for scanning transmission electron microscopy (STEM). In particular, accuracy and precision in STEM is significantly hampered by the presence of sample drift and scan distortion. While sample drift is usually the dominate consideration, the scan system calibration is rarely perfectly orthonormal, which can introduce significant errors. These limitations have obscured the capabilities to directly characterize minute atomic structure changes that can ultimately control properties.

In this talk, we will show how revolving scanning transmission electron microscopy (RevSTEM) is able to achieve highly accurate imaging on a routine basis [1]. The method uses a series of fast-acquisition STEM images, but with the scan coordinates rotated between successive frames. This, however, only accounts for the drift introduced distortion. Equipped with the Atom Column Indexing approach [2], we will introduce a method to very accurately and precisely correct the global scan coordinate system, to ensure a near-perfectly orthonormal scan. We will demonstrate the stability of this correction and that the combination of RevSTEM with such a corrected scan system is capable of achieving sub-picometer accuracy of real-space crystallographic measurements in STEM [3].

Multiple case studies will be presented to demonstrate the power of this new technique in combination with density functional theory. For example, we will show how picometer precise measurements enable the capability to directly observe static atomic displacements within a complex oxide and chalcogenide solid solutions [3, 4]. These subtle distortions will be examined using density functional theory to investigate the role of local chemical fluctuations and the influence of bond strengths. With the level of accuracy afforded by this imaging approach we will show how concepts used in analyzing total diffraction data, such as the pair distribution function (PDF), can be applied to STEM data. To do so, we will introduce the projected-PDF to analyze correlation between displacements spanning the entire image [4]. Furthermore, we will also show how RevSTEM images can be used to accurately determine crystallographic parameters in real-space for ferroelectric $\mathrm{HfO}^{2}$ thin films and to investigate local chemical ordering in high entropy alloys $[5,6]$.

Finally, we will emphasize that it is becoming possible to quantify all aspects of our electron microscopy data for a rich set of comparisons between theory and experiment. Recently developed methods seeking to disrupt previously accepted paradigms, for example, have now overcome limitations of STEM to enable for a new level of comparison between local atomic distortions through a combination of theory and experiment [7]. These results open a new world of atomic scale exploration that was previously just beyond our reach.

References:

[1] X Sang and JM LeBeau, Ultramicroscopy 138 (2014), 28-35. 
[2] X Sang, AA Oni, A. A., and JM LeBeau, Microscopy and Microanalysis 20 (2014), 1764-1771.

[3]. JH Dycus et al, Microscopy and Microanalysis 21 (2015), 946-952.

[4] X Sang et al, Applied Physics Letters 106 (2015), 061913.

[5] X Sang et al, Applied Physics Letters 106 (2015), 162905.

[6] C Niu et al, Applied Physics Letters 106 (2015), 161906.

[7] AB Yankovich et al, Nature Communications 5 (2014), 1-7.

[8] The authors acknowledge funding from the Air Force Office of Science Research (FA9550-14-1-

0182 and FA9550-12-1-0456) and the National Science Foundation (DMR-1350273).

(a)

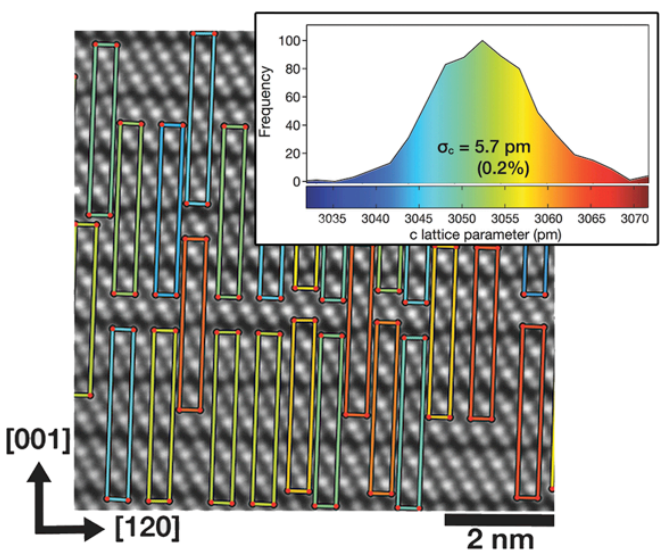

(b)

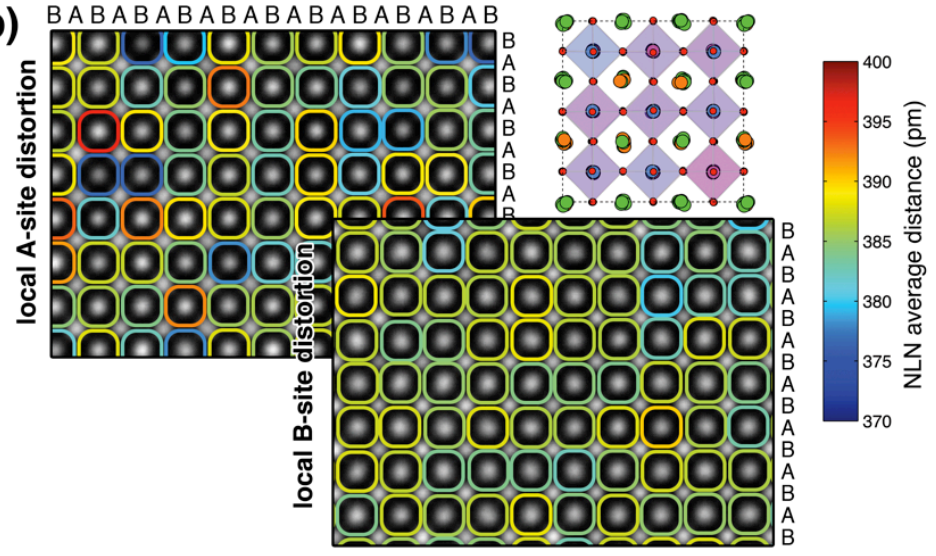

Figure 1. (a) High-angle annular dark-field scanning transmission electron microscopy (HAADF STEM) image and histogram of c lattice parameters (inset) of pure $\mathrm{Bi}_{2} \mathrm{Te}_{3}$ along $<100>$. The color of each rectangle corresponds to the color scale in the in-set. (b) Average A-A ( $\mathrm{La} / \mathrm{Sr}$ ) and B-B (Al/Ta) nearest like neighbor distances around each sub-lattice atom column of a $\left(\mathrm{La}_{0.18} \mathrm{Sr}_{0.82}\right)\left(\mathrm{Al}_{0.59} \mathrm{Ta}_{0.41}\right) \mathrm{O}_{3}$ single crystal.
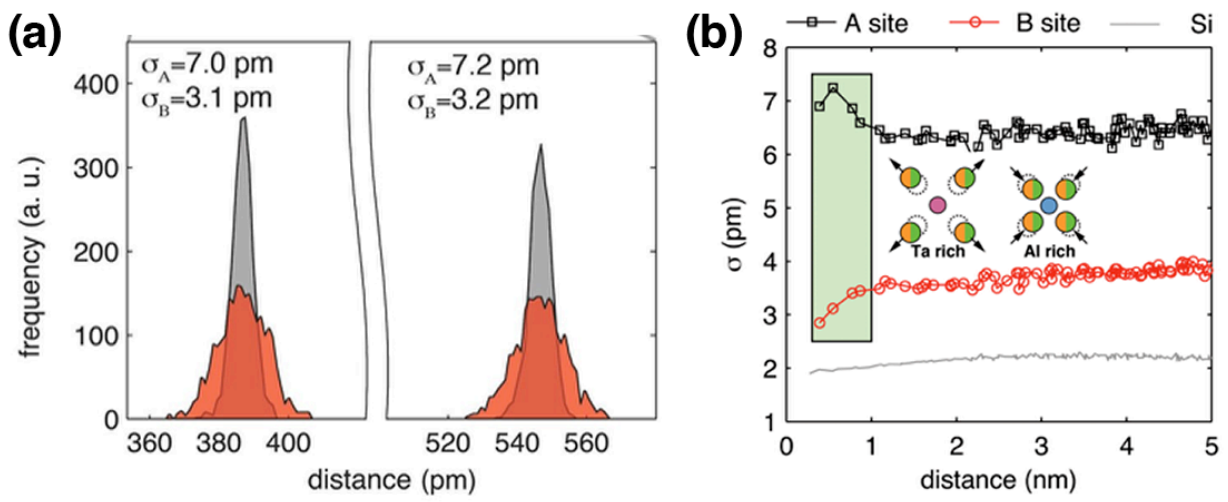

Figure 2. The projected pair distribution function for A-La/Sr (red) and B-Ta/Al (gray) sub-lattices calculated based on $\mathrm{n}^{\text {th }}$ like-neighbor atom columns. Standard deviation of the atom column pair distances for A (black) and B (red) sub-lattices compared to that from Si (line). Inset unit cells explain the origins of the increased standard deviation for near neighbors on the B sub-lattice. 